\title{
ESSENTIAL NORM OF THE DIFFERENTIAL OPERATOR
}

\author{
Tesfa Mengestie
}

Abstract. This paper is a follow-up contribution to our work [10] where we studied some spectral properties of the differential operator $D$ acting between generalized Fock spaces $\mathscr{F}_{(m, p)}$ and $\mathscr{F}_{(m, q)}$ when both exponents $p$ and $q$ are finite. In this note we continue to study the properties for the case when at least one of the spaces is growth type. We also estimate the essential norm of $D: \mathscr{F}_{(m, p)} \rightarrow \mathscr{F}_{(m, q)}$ for all $1 \leqslant p, q \leqslant \infty$, and showed that if the operator fails to be compact, then its essential norm is comparable to the operator norm and $\|D\|_{e} \simeq\left|m^{2+p}-m^{1+p}\right|^{\frac{1}{p}} \simeq\|D\|$.

Mathematics subject classification (2010): 47B32, 30H20, 46E22,46E20,47B33.

Keywords and phrases: Generalized Fock spaces, essential norm, norm, bounded, compact, differential operator, composition operator.

\section{REFERENCES}

[1] M. J. Beltrán, Dynamics of differentiation and integration operators on weighted space of entire functions, Studia Matematica 221 (1), (2014), 35-60.

[2] H. Bommier-HatoA, M. Englis, And El-HASs An Youssfia, Bergman-type projections in generalized Fock spaces, J. Math. Anal. Appl. 389 (2), (2012), 1086-1104.

[3] A. Borichev, R. Dhuez And K. Kellay, Sampling and interpolation in large Bergman and Fock spaces, J. Funct. Anal. 242, (2007), 563-606.

[4] O. CONSTANTIN AND ANn-MARIA PERSSOn, The spectrum of Volterra-type integration operators on generalized Fock spaces, Bull. London Math. Soc. 47 (6), (2015), 958-963.

[5] O. Constantin And José Ángel PelÁEZ, Integral Operators, Embedding Theorems and a Littlewood-Paley Formula on Weighted Fock Spaces, J. Geom. Anal., 26, 2 (2016), 1109-1154.

[6] Z. CUCKOVIĆ AND R. ZHAO, Weighted composition operators between different weighted Bergman spaces and different Hardy spaces, Illinois J. Math 51, (2007), 479-498.

[7] Z. CuCKOvić AND R. ZHAO, Weighted composition operators on the Bergman space, J. London Math. Soc. 70, (2004), 499-511.

[8] A. HaRUTyUnyan, W. Lusky, On the boundedness of the differentiation operator between weighted spaces of holomorphic functions, Studia Math. 184, (2008), 233-247.

[9] D. LUECKING, Embedding theorems for space of analytic functions via Khinchine's inequality, Michigan Math. J. 40, (1993), 333-358.

[10] T. Mengestie, A note on the differential operator on generalized Fock spaces, J. Math. Anal. Appl. 458 (2), (2018), 889-1708.

[11] T. Mengestie And S. UEKI, Integral, differential and multiplication operators on weighted Fock spaces, Complex Anal. Oper. Theory. https ://doi .org/10.1007/s11785-018-0820-7.

[12] T. Mengestie, Carleson type measures for Fock-Sobolev spaces, Complex Anal. Oper. Theory 8, (2014), 1225-1256.

[13] T. Mengestie, On The Spectrum of Volterra-Type Integral Operators on Fock-Sobolev spaces, Complex Anal. Oper. Theory., https://doi .org/10.1007/s11785-016-0629-1.

[14] T. Mengestie, Spectral properties of Volterra-type integral operators on Fock-Sobolev spaces, J. Korean Math. Soc., https ://doi .org/10.4134/JKMS.j160671.

[15] V. L. OLEINIK, Embedding theorems for weighted classes of harmonic and analytic functions, J. Math. Sci. 9 (2), (1978), 228-243. 
[16] J. PAU AND J. A. PELÁEZ, Embedding theorems and integration operators on Bergman spaces with rapidly decreasing weightes, J. Funct. Anal. 259 (10), (2010), 2727-2756.

[17] J. RÄTTY Ä, The essential norm of a composition operator mapping into the $Q_{s}$-space, J. Math. Anal. Appl. 333, (2007), 787-797.

[18] J. SHAPIRO, The essential norm of a composition operator, Annals of Math. 125, (1987), 375-404.

[19] Stevo Stević, Weighted composition operators between Fock-type spaces in $\mathbb{C}^{N}$, Appl. Math. Comput. 215, (2009), 2750-2760.

[20] S. I. UEKI, Weighted composition operators on some function spaces of entire functions, Bull. Belg. Math. Soc. Simon Stevin 17, (2010), 343-353. 